\title{
AUTOTOMÍA CAUDAL COMO ESTRATEGIA GERENCIAL PARA EL CAMBIO ORGANIZACIONAL EN EMPRESAS FAMILIARES ${ }^{1}$
}

\section{CAUDAL AUTOTOMY AS A MANAGERIAL STRATEGY FOR ORGANIZATIONAL CHANGE IN FAMILY BUSINESSES}

\author{
Omar El Kadi ${ }^{2}$, Cira de Pelekais ${ }^{3}$, Miguel Ángel Robles ${ }^{4}$ y Martín Leal ${ }^{5}$ \\ Universidad Privada Dr. Rafael Belloso Chacín, URBE. Maracaibo (Venezuela)
}

RECIBIDO: 2 de octubre de 2014

ACEPTADO: 10 de diciembre de 2014

\section{RESUMEN}

Este artículo tuvo como objetivo analizar la autotomía caudal como estrategia gerencial para el cambio organizacional en empresas familiares del municipio Maracaibo del estado Zulia. Se definió como un estudio empírico-analítico-positivista de tipo analítico, explicativo no experimental, transeccional y de campo. Se diseñaron dos (2) instrumentos, uno para cada variable y aplicado a una población de treinta (30) gerentes en ocho (8) entidades; dichos cuestionarios tuvieron treinta y tres ítems para la variable autotomía caudal y cuarenta y dos (42) para cambio organizacional, ambos con cinco alternativas de escala tipo Likert. Su validez fue confirmada por siete (7) expertos en el área y luego se pasó una prueba piloto en doce (12) sujetos que representan la muestra de la población donde finalmente se aplicó. Seguidamente se realizó el cálculo del coeficiente Alpha Cronbach, y se obtuvo como resultado 0.864, que es altamente confiable. Así, queda en evidencia que en las etapas de transformación la "situación amenaza" es el primer proceso que enfrentan los gerentes en el proceso de transformación, seguido de la "renovación", "dolor" y "desprendimiento". Se concluye que las etapas de transformación en los contextos estudiados, la situación amenaza forma parte de los aspectos del ambiente que pueden llegar a constituir un peligro para el logro de los objetivos de las empresas familiares, aun cuando se considera un acto social o fenómeno natural que se manifiesta en el entorno de ellas mismas.

Palabras clave: autotomía caudal, cambio organizacional, empresas familiares, desprendimiento, transformación.

\begin{abstract}
This article aimed to analyze the flow autotomy as a management strategy for organizational change in family businesses in the city of Maracaibo, Zulia state. It was defined as an empirical, analytical UN and positivist study of the analytic type, explanatory non-experimental, trans-sectional and field. Two (2) Instruments were designed for each of the variables Each paragraph is designed and implemented for a population of thirty (30) Managers in eight (8) Entities; these Questionnaires had thirty-three items for each caudal autotomy variable and forty-two (42) Organizational Change paragraphs, both with five alternatives of the Likert scale. Its validity was conducted by seven (7) experts in the field and then underwent a pilot test to twelve (12) individuals who represent the sample of the population where he eventually applied. The reliability thereof is performed by calculating the Cronbach Alpha coefficient, resulting in 0.864 which is highly reliable. The results show that in the stages of transformation the "Status Threat "is the first trial facing managers in the transformation process, followed by the "Renewal", "Pain" and "Detachment" . Concluding that the stages of transformation in the contexts studied, the situation threatens, is part of the environmental aspects that may constitute a danger for the achievement of the objectives of family businesses, considered a social event or a natural phenomenon, manifested in the vicinity thereof.
\end{abstract}

Key words: Caudal Autotomy, Organizational Change, Family Businesses, Detachment, Transformation

Este artículo se puede referenciar:

El Kadi, O.; Pelekais, C.; Robles, M. \& Leal, M. (2015). Autotomía caudal como estrategia gerencial para el cambio organizacional en empresas familiares. En Desarrollo Gerencial. Revista de la Facultad de Ciencias Económicas, Administrativas y Contables, 7(1). 68-84.

\footnotetext{
${ }^{1}$ Proyecto de Investigación: Autotomía caudal como estrategia gerencial para el cambio organizacional en empresas familiares

2oelkadi@urbe.edu.ve

3 cirapelekais@urbe.edu

4 Miguel.robles@urbe.edu.ve

5 lealmartin@hotmail.com
}

Desarrollo Gerencial, 7 (1) pp. 68 - 84 Enero-Junio 2015. ISSN: 2145-5147 (On Line). Universidad Simón Bolívar. Barranquilla-Colombia. Contactos: desarrollogerencial@unisimonbolivar.edu.co 


\section{1.- INTRODUCCIÓN}

Es muy posible que en las ciencias gerenciales se hayan experimentado algunos episodios en los cuales el riesgo que toleran a diferentes eventos, hace que algunos integrantes de una organización generen su propio miedo ante la amenaza de ser sustituido por otra persona, por la tecnología o simplemente por los cambios que trae la aplicación de estrategias de mercado, lo que constituye una de las fuerzas selectivas más influyentes, por cuanto se concibe en términos naturales como depredación.

En procesos gerenciales y en una especie de consiliencia como disposición voluntaria de unir los conocimientos de la biología y la información de la disciplina gerencial, creando un marco unificado de entendimiento, se observa que la misma necesidad de evitar ser "depredado" dentro de una organización mientras se atiende a otras actividades internas, ha favorecido la evolución de una variedad de estrategias antidepredatorias con gran flexibilidad de comportamientos de los individuos, cuya habilidad puede estar relacionada con la capacidad de estimar los niveles de riesgo de depredación y de generar cambios.

Ante este planteamiento, el escape de un depredador mediante alguna táctica es común entre muchos animales, y en este caso de los reptiles, de quienes se toma su comportamiento de la autotomía caudal como proceso. La decisión de cuándo y cómo escapar es crucial, y es de esperar que haya sido optimizada por la selección natural, teniendo en cuenta que el huir tiene, aparte de los beneficios obvios de escapar, una serie de costos, razón por la que existiría una distancia óptima de huida entre la situación depredadora y el individuo mismo como presa. En este sentido, las observaciones y experimentos realizados en reptiles se ajustan a las predicciones: si se incrementan los costos de huida, la distancia disminuye, mientras que cuando se incrementa el riesgo de ser capturado, la distancia de huida aumenta para generar un cambio.

Por ello, la aplicación de estrategias ocasiona algún impacto en la organización y es una relación entre un proceso natural y la ciencia social de la gerencia, que hasta ahora ha sido ignorada como estudio comparativo sobre ecología en relación con el comportamiento de los reptiles y el comportamiento humano en una organización. Sin embargo, al llevarlo a este campo, el riesgo de depredación es un factor esencial para una comprensión completa de la ecología, comportamiento y evolución de la vida de los individuos en una entidad donde hacen su labor. En este orden de ideas, el estudio de los mecanismos antidepredatorios de los reptiles puede contribuir al avance de la ciencia gerencial en virtud de que permite establecer teorías generales y comprender los límites de la generalidad.

Así, este artículo abre un compás a la observación mostrando algunos indicios a futuras investigaciones y sustenta la teoría de efecto autotómico caudal para lograr cambios organizacionales, mediante una presentación donde los autores hacen la inferencia interpretativa de los comportamientos de lagartijas 


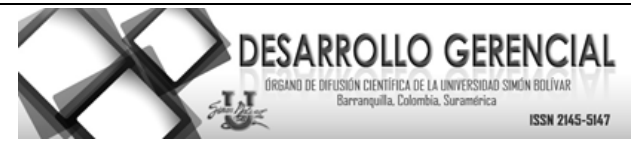

según las actitudes gerenciales que, en un momento determinado, llevan a proponer una nueva significancia sobre los tipos de gerentes que se distinguen en la actualidad.

\section{Bases teóricas}

En Venezuela, son muy incipientes los estudios realizados en el área de la autotomía caudal, sobre todo lo que con respecto al área organizacional signifique inferir la aplicación del término biológico en los contextos de transformación estructural, funcional o estratégica en el ámbito del comportamiento humano, pudiéndose decir con propiedad que no existen referentes en las universidades del país, a excepción de unos trabajos realizados a nivel de investigaciones en roedores suramericanos y que pueden traspolarse analógicamente a contextos organizacionales. No obstante, se han hecho investigaciones sobre la variable cambio organizacional que se ocupan de la evaluación de puestos de trabajo en diferentes esferas de las organizaciones tanto en términos generales como específicos. Sin embargo, cruzando las dos (2) variables, no pudo ubicarse ningún trabajo, artículo o ponencia expuesto en eventos científicos.

En cambio, fueron ubicadas prolijas investigaciones referenciadas internacionalmente sobre la variable cambio organizacional, las cuales contribuyeron a incrementar el entendimiento de las planteadas como objeto de estudio, y entre las que se encuentran las de Albornoz (2009) en su trabajo titulado: Cultura y cambio organizacional en empresas de telefonía móvil. El investigador se propuso determinar la relación entre la cultura y el cambio organizacional en las empresas del sector de telefonía móvil del municipio Maracaibo (estado Zulia), con lo que facilitó a este estudio información sobre el cambio organizacional, y especialmente sobre las fuentes de resistencia.

También se consultó el trabajo de Morales (2007), cuya investigación Cambio organizacional y estrés laboral en los gerentes de las empresas regionales de televisión por cable tuvo como propósito determinar la relación entre ambos aspectos basándose en postulados de Robbins (2004). De esta manera sirvió como soporte al presente estudio en la medida en que demuestra que el cambio organizacional está representado por un conjunto de alteraciones tanto estructurales como de comportamiento que no ocurre espontáneamente, sino por la presión de fuerzas externas y fuerzas internas.

\section{Consiliencia de la autotomía caudal como estrategia gerencial}

Cualquiera sea la fundamentación de una organización, es decir: al margen de que su estructural y normativa sea lucrativa, o de beneficio netamente social (sin fines de lucro), esta debe contar con una serie de estrategias que canalicen el logro de los objetivos propuestos, según la guía de un plan estratégico o situacional para el desarrollo armonioso de su cometido. En este sentido, las estrategias que siga una 
organización deben estar referidas a la unificación de criterios y la cohesión de opiniones y decisiones individuales a fin de misionar en conciliación un trabajo en equipo que le represente a la entidad los mejores beneficios, así como a sus miembros.

Entonces se puede señalar que las concepciones de las estrategias gerenciales, además del trabajo en equipo, se fundamentan en el liderazgo y el servicio, punto clave de transformación enmarcado dentro de los aportes realizados por los investigadores; a lo largo del trabajo, dicho eje fue necesario con el objeto de asimilar de la autotomía caudal lo novedoso que ofrece la naturaleza para su aplicación. De acuerdo a lo explicado por Senge (2000), la transformación como condición es el reconocimiento de que toda la organización está en permanente evolución; tan es así que la dinámica de los procesos no se detiene, y permite valorar la necesidad urgente de asumir la diferencia como parte de la gestión.

Se destaca que la transformación posibilita evolucionar y dimensionar la gestión; pretender quedarse en el pasado es renunciar a la realidad planteada por el mercado. Es común encontrar semánticamente la unión entre cambio y transformación, y evidentemente muchas organizaciones le apuestan más al primero que al segundo; sin embargo, lo natural de los procesos es el debido tránsito de un estado a otro. En este sentido, el cambio se usa cuando en efecto se trata de experiencias cualitativas conducentes a la aparición o incorporación de nuevos elementos para reemplazar a otros.

De acuerdo con los postulados expuestos en el libro la Quinta disciplina, los cambios en las organizaciones son necesarios por cuanto a estas las constituyen masas sociales que son cambiantes, según lo exija el requerimiento de un nuevo talento humano. En cuanto al proceso autonómico referido como un hecho natural de desprendimiento, que es alimentado en un ambiente que soporta la subsistencia en medio de las adversidades, ofrece oportunidades grandiosas de crecimiento.

De igual forma, Dempsey (2009) extrapola algunos elementos propios de este tipo de animal para realizar una analogía con los hábitos gerenciales puestos de manifiesto por la población estudiada; a tal efecto se sugiere revisar la tabla 1 que a continuación se detalla:

Tabla 1. Hábitos gerenciales

\begin{tabular}{|c|c|c|c|c|c|}
\hline Factor/Tipo & $\mathbf{1}$ & $\mathbf{2}$ & $\mathbf{3}$ & $\mathbf{4}$ & $\mathbf{5}$ \\
\hline Lagartija 1 & Ser psicótico & $\begin{array}{c}\text { Ser } \\
\text { emocional }\end{array}$ & Alucinar & Ser franco & Ser obsesivo \\
\hline Lagartija 2 & Ser un llorón & $\begin{array}{c}\text { Ser } \\
\text { pesimista }\end{array}$ & Ser optimista & Ser adulador & $\begin{array}{c}\text { Estar } \\
\text { preocupado }\end{array}$ \\
\hline Lagartija 3 & $\begin{array}{c}\text { Ser un conocedor } \\
\text { de todo }\end{array}$ & $\begin{array}{c}\text { Perder la } \\
\text { memoria }\end{array}$ & $\begin{array}{c}\text { Nutrirse de } \\
\text { cosas positivas }\end{array}$ & $\begin{array}{c}\text { Cometer } \\
\text { errores }\end{array}$ & No Conservar \\
\hline Lagartija 4 & Enojarse & $\begin{array}{c}\text { Molestar a la } \\
\text { gente }\end{array}$ & Ser narcisista & Ser impulsivo & Ser infantil \\
\hline Lagartija 5 & $\begin{array}{c}\text { Ser co- } \\
\text { dependiente }\end{array}$ & $\begin{array}{c}\text { Ser pícaro } \\
\text { Ser un tirano }\end{array}$ & $\begin{array}{c}\text { Ser un } \\
\text { procrastinado }\end{array}$ & Ser un copiador \\
\hline
\end{tabular}

Desarrollo Gerencial, 7 (1) pp. 68 - 84 Enero-Junio 2015. ISSN: 2145-5147 (On Line). Universidad Simón Bolívar. Barranquilla-Colombia. Contactos: desarrollogerencial@unisimonbolivar.edu.co 


\begin{tabular}{|c|c|c|c|c|c} 
Lagartija 6 & Ser engreído & $\begin{array}{c}\text { Ser un } \\
\text { comediante }\end{array}$ & Ser voluble & $\begin{array}{c}\text { Ser un } \\
\text { controlador }\end{array}$ & $\begin{array}{c}\text { Limitar el } \\
\text { vocabulario }\end{array}$ \\
\hline
\end{tabular}

Fuente: Interpretación y adaptación propia (2014), según Dempsey (2009).

\section{Implicaciones del efecto autotómico organizacional desde la perspectiva que ofrece el campo de las ciencias administrativas y gerenciales}

Tomando la concepción organizacional como parte de un sistema que, a su vez, desarrolla otro subsistema, Prigogine (1993) enmarca esta condición de sistema y subsistema en el contexto de las ciencias humanas a las que determina por las incertidumbres de lo que ha de pasar o acontecer mientras se logra una determinada meta u objetivo; para la prefijación de propósitos se emplean diferentes recursos de naturaleza distinta, con lo que se reconoce la manifestación ontológica del sentido que se posee de un sistema con sus variables, las cuales se dimensionan para interactuar entre sí y fijar una interrelación de retroalimentación constante bajo un orden preestablecido para su total aceptación y comprensión.

No obstante, también se reconoce que estas entidades forman parte de otros sistemas no estáticos, que afectan y son afectados por estos, bajo una perspectiva fractal; son representados por ejemplo, en el departamento, la empresa, industria, sociedad y cultura, así como en infraestructura, servicios e instituciones de apoyo; políticas macroeconómicas, país, continente. Aunque permanezcan en la escena, no necesariamente responden de la misma manera con el paso del tiempo a consecuencia de su naturaleza dinámica.

Conforme a lo sustentado por Etkin (2009), las organizaciones deben ser vistas desde la perspectiva del diseño, su construcción social y la manifestación de esquemas y relaciones estables en el tiempo para hacer previsibles y considerarlas como válidas cuando se manifiestan en otras situaciones con los mismos actores u otros o distintos, bajo las mismas condiciones.

De lo anterior se desprende la necesidad de cohesionar esfuerzos en un ambiente de complejidades que son auspiciadas por el dinamismo y en donde coexisten una gama de fuerzas que pueden ser atractores organizacionales, que Cornejo (2004) define como el conjunto de variables y elementos internos que se dimensionan en una actividad de connotación estratégica para explicar lo sucedido, en un momento determinado coinciden y otros se alejan; en ocasiones emergen sistemas estables y en otros inestables, por lo que el comportamiento organizacional puede ser visto y estudiado como un sistema caótico.

En concordancia con los planteamientos anteriores, Morin (2007) señala que los conocimientos humanos son simples; por consiguiente, es poco el beneficio que ofrecen a los efectos de conocer las características de la organización: un todo es más que la suma de las partes que lo constituyen; seguidamente, como se cuenta con la noción de organización, entonces se dificulta tratar de explicar una 


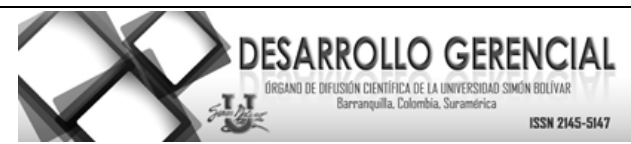

parte de esta, puesto que el todo es, entonces, menos que la suma de las partes. Así resulta compleja su comprensión: "El todo es más y al mismo tiempo menos que la suma de las partes".

Por lo anterior, y según señalamientos de Schvarstein (1998), es difícil explicar estos sistemas sociales por "alguna ley simple", sobre todo cuando en las condiciones iniciales se hacen presentes las tensiones: modelos sostenidos por la alianza entre las ciencias puras y las ciencias sociales, entre la vida biológica y la vida social, tal como lo explica Morin (2007). De esta de manera, la aplicación de la teoría de caos y la complejidad como un nuevo paradigma emergente constituye una herramienta útil para tales propósitos.

Vale la ocasión para resaltar que lo postulado en la teoría de la autoorganización solo se vinculaba a la cibernética según señalamientos de Morin (2007); sin embargo, fue propuesta como fundamento de la comprensión de la ciencia al tratar de explicar el proceso de los seres vivos. Desafortunadamente tales explicaciones se disiparon para abrir a otros entendimientos como el hecho de comprender de manera lógica y coherente lo que sucede con la identificación de las situaciones que se suscitan en los sistemas caóticos al observar sus variables de manera directa en su entorno y delimitar sus elementos atractores, para así establecer las relaciones que facilitan el planteamiento de ecuaciones matemáticas con un sentido de comprobación.

Sumado a lo anterior, el mismo autor ya prenombrado indica que al observar los aspectos condicionantes de las situaciones de un sistema caótico, se pueden determinar los elementos atractores desde una perspectiva autonómica una vez se identifican los factores condicionantes de un determinado comportamiento en una organización como sistema. Obviamente, a esta determinación se le añade la búsqueda de comprensión de los porqués de determinado comportamiento, es decir, cuáles son las causas que lo originan hasta convertirse en un objeto geométrico como parte constitutiva de un todo que se repite a distintas escalas, en una dinámica natural establecida por reglas que incluyen un orden que se desordena como resultado del caos, pero que tiene una explicación y razón de ser para la supervivencia o mantenimiento ante nuevos contextos.

\section{Autotomía caudal}

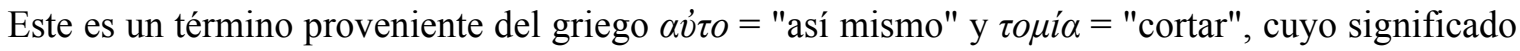
es automutilación que generalmente lo hace un animal en una parte o apéndice del cuerpo para luego adaptarse al medio ambiente donde habita, siendo un acto que realiza en algún momento ante la amenaza que siente por diversos factores del medio y lo hace para huir y evitar ser depredado. De acuerdo a lo explicado por Zani et al. (2013), es un proceso de naturaleza propia de los invertebrados, que bien pueden auto-mutilarse la cola, las patas, antenas o cualquier otra parte de su estructura ya ornamental o defensiva con el fin subsistir. 
Desde esta perspectiva fisiológica y celular, es un proceso que experimentan los reptiles desde sus ancestros: los anfibios, para regenerar parte de sí comenzando por una adaptación transformacional hasta que logran el cambio requerido y adquieren las habilidades necesarias de su nueva vida. Cabe destacar que los estudios realizados por Sagarin Zani, Russel \& (2013) señalan que este episodio se caracteriza por situaciones de riesgo a las que se somete el animal de manera fortuita, o cuando algunas aves como las águilas experimentan una especie de renacer.

\section{Etapas de transformación}

Cuando se mira el cambio, no se puede obviar la pérdida; por lo tanto, la esencia del cambio es la pérdida. Según señala Dempsey (2009), con cada cambio algún tipo de pérdida ocurre, y cuando se piensa en cambios en los acontecimientos cotidianos de las vidas de las personas, con motivo de trabajo, enfermedad, matrimonio, divorcio, o mudanza, se debe pensar: ¿Cuáles eran las pérdidas? En tales casos, se presenta una versión lógica de los hechos:

La génesis del duelo empieza con las necesidades dobles de aferrarse y desprenderse, posiblemente de un bebé que no ha nacido, se diría que está viviendo una vida de gozo; sin preocupaciones de ningún tipo, pues todas sus necesidades están cubiertas, no tiene cuentas por pagar, ni un jefe que satisfacer, su vida es de pleno disfrute. Después de 9 meses, otra realidad llega, y traumáticamente separa al bebé de este ambiente sereno para traerlo a un ambiente más frío y cruel. Ante situaciones diversas el individuo experimenta etapas de transformación por alguna situación de amenaza, desprendimiento, dolor y renovación.

\section{Proceso del desprendimiento}

Muchos aspectos de la autotomía caudal han sido revisados, tales como su distribución sistemática detallada y mecanismos histológicos que señalan las explicaciones de Bellairs y Bryant (1985), la historia evolutiva de Arnold (1994), y aspectos ecológicos y de comportamiento de este mismo autor (1988). Se conocen numerosos efectos en el proceso de la pérdida de la cola de los animales que experimentan este fenómeno. Sin embargo, de la mayoría de los casos se tiene alguna referencia a partir de experimentos en laboratorio.

Se destaca que los mecanismos de autotomía en una especie determinada de saurio solamente pueden ser mantenidos si son adaptativos. Por tanto, aquellos que han perdido la cola deberían ser capaces de hacer frente a las desventajas de su condición, para lograr sobrevivir durante el periodo de regeneración y 


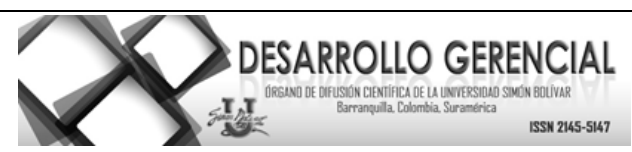

transmitir sus genes a las generaciones futuras, experimentando rotura por el desprendimiento, adaptación a la nueva situación, aprovechamiento de las reservas y la consiguiente regeneración.

\section{Efecto estratégico}

A comienzos de los años sesenta, científicos interesados por los cambios climáticos intentaron encontrar un modelo matemático para explicar el comportamiento de la atmósfera y hacer predicciones a partir de variables sencillas. Uno de ellos fue Lorenz (2000), quien planteó un sistema no lineal de tres ecuaciones diferenciales, reconocidas como las ecuaciones de Navier-Stokes; con tres variables, logró presentar un modelo ajustado sobre las variaciones de la velocidad y la temperatura del aire a lo largo del tiempo. El modelo publicado se expresó en los siguientes términos:

$$
\begin{aligned}
& X=(Y-X) \\
& Y=r X-Y-X Z \\
& Z=X Y-b Z
\end{aligned}
$$

\section{Dónde:}

$\mathrm{X}=$ razón de rotación del sistema

$\mathrm{Y}=$ gradiente de temperatura

$\mathrm{Z}=$ desviación de la temperatura

$\mathrm{a}=$ número de Prandtl (viscosidad, conductividad térmica)

$\mathrm{r}=$ diferencia de temperatura entre la base y el tope del sistema, denotado como número de Rayleigh.

$\mathrm{b}=$ razón entre la longitud y altura del sistema, es la relación de aspectos de los rollos o recirculaciones por convección.

Luego de algunas iteraciones Lorenz (2000) observó que pequeñas variaciones en las condiciones iniciales del sistema, como, por ejemplo, utilizar 3 en lugar de 6 decimales, llevaba a grandes diferencias en los escenarios futuros, que generaba una ruptura en la manera tradicional de concebir el comportamiento de los sistemas, incluso dificultando las predicciones meteorológicas a largo plazo, las cuales modifican su ruta desde estados deseados hacia estados reales. Y aun cuando las perturbaciones son pequeñas, traen implicaciones importantes en el resultado final como mecanismo de defensa eficaz, reingeniería y funcionalidad hacia la optimidad.

\section{Cambio organizacional}

Para Ponce (2002), el cambio organizacional se manifiesta en la adaptación de las organizaciones a las variantes condiciones del medio ambiente externo o interno mediante procesos de aprendizaje. La 
capacidad para el cambio es lo que caracteriza a las organizaciones exitosas. El cambio surge como consecuencia de interacciones de fuerzas tanto internas como externas. En este sentido, Kinicki y Kreitner (2003) plantean que el ritmo de cambio organizacional y social se está acelerando claramente y que las compañías deben cambiar para sobrevivir, pues de su adaptación dependerá el logro de las metas y el éxito alcanzado en un largo plazo.

Por su parte, Davis y Newstrom (2003) refieren que el cambio organizacional es toda modificación que ocurre en el ambiente de trabajo y afecta la manera en que deben actuar los empleados. El proceso de cambio es muy complejo y en él influyen diversos factores dentro de la organización; además, es preciso abordarlo hacia todos los niveles, funciones y acciones dentro de ella misma. Por esta razón, el cambio planeado no solo lo debe llevar a cabo un agente, sino que debe ser una filosofía de trabajo para todos los miembros de la empresa.

De igual forma, Koontz y Weihrich (2004) explican que los trabajadores perciben de diversa forma los cambios organizacionales. Esta percepción incluye un análisis personal de las situaciones que constituyen innovaciones necesarias con el apoyo del sistema total de la y en correspondencia con sus objetivos. Por ello los mismos autores aseveran que en el proceso de administración del cambio es necesario identificar las fuentes de conflicto e identificar la resistencia al cambio.

También Pelekais y Soto (2014) reseñan que al plasmar el tema de cambio es necesario referirse primeramente a la etimología de la palabra, la cual significa junto a la vida; por lo tanto, los cambios en todos los contextos son vida y necesidad. Una organización requiere producir, para eso se creó, y la única forma de hacerlo es manteniéndose viva a través de sus actualizaciones estructurales, estrategias, profesionalización del equipo de trabajo y, por ende, la humanización de ese equipo. Solo integrando estos elementos se puede hablar de cambio organizacional.

Hoy en día se conjugan elementos complejos y transdisciplinarios a nivel organizacional que provocan transformaciones, reinvención y orden de una corporación. Se puede decir que un orden + caos por los factores psicosociales que están aflorando en escenarios globales de incertidumbre. Sin embargo, en tiempos de crisis superviven los más aptos y adaptables, por ello, el cambio aviva, apasiona y sigue la idea generada por un talento humano que inteligentemente impulsa herramientas innovadoras para que la organización se diferencie y supere a otras en épocas caóticas siempre que se transforme y sea la mejor en su unidad de negocio.

\section{Tipos de fuerzas}

Existen indicios que marcan la necesidad de cambio y los mismos se verifican vigilando las fuerzas que este trae. En dicho proceso las organizaciones enfrentan a estas corrientes, algunas de las cuales provienen 


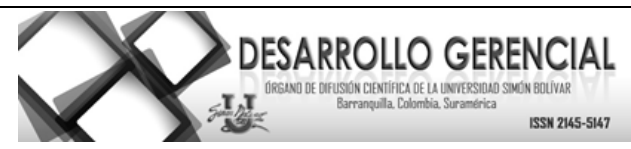

del entorno de las empresas, por lo que se identifican como externas, o si están dentro de la organización se les califica como internas. En este sentido, Guizar (2004) afirma que el cambio responde a diversidad de factores entre los cuales se encuentran tanto variables propias del ambiente de las organizaciones como externas a la misma, así como fuerzas externas e internas donde se denotan adelantos tecnológicos, cambios de mercados y presiones sociopolíticas en las primeras mientras en las segundas subyacen la gestión de personal y la acción directiva.

\section{Fuentes de resistencia}

Uno de los resultados mejor documentados de los estudios sobre el comportamiento de los individuos y organizaciones es que los individuos ofrecen resistencia al cambio. Sobre el particular la definición de Gordon (2000) señala que ello incluye una postura actitudinal contraria del trabajador, en cuanto a las transformaciones que propone la organización a la que pertenece, como son las medidas alternas dirigidas hacia la mayor productividad de la misma. De igual manera Koontz y Weihrich (2004) sostienen que la resistencia al cambio a nivel organizacional es un comportamiento inevitable al mismo tiempo que no hay argumento alguno para tomarlo como un fenómeno social, porque se fundamenta en fenómeno psicológico de la resistencia; ahí se reconoce la fuente individual denotada por los hábitos, los factores económicos y miedo a lo desconocido. $\mathrm{Y}$ en cuanto a la fuente organizacional, se designa por enfoques limitados al cambio y las amenazas.

\section{Requerimientos}

Las organizaciones a lo largo de los años han sido consideradas como espacios para la producción con mano de obra calificada, donde laboran grupos de personas que interactuando en determinados sitios dotados de maquinaria o herramientas específicas, obtienen un determinado bien o servicio. No obstante, las personas están en una institución, y en ese escenario interactúan entre pares que de manera directa o indirecta propician acciones importantes, y por esa razón deben ser tomadas en cuenta.

Al respecto Benavides Espindola (2001) expresa que, precisamente por ser de carácter clave, el desarrollo de una organización es diferente al buscar un cambio gestionado, aquellas compañías que se encuentran en la etapa de transición o cambio, poseen un punto clave, al reconocer que las habilidades forman parte del planteamiento de transformación y el fruto de progreso en la práctica. Por ello, de la misma manera que las empresas familiares deben tener la capacidad de decidir sobre los proyectos de cambio de acuerdo con sus propias prioridades, también tienen que existir oportunidades de trabajar juntos para el desarrollo del cambio organizacional, así como los recursos adecuados. Entre ellos figuran el 


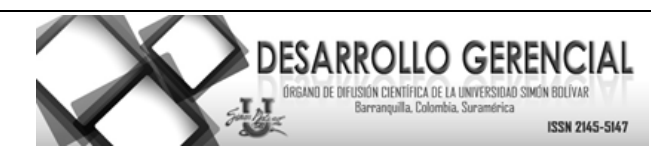

requerimiento personal de la formación y la participación; así como en el plano colectivo el afianzamiento del liderazgo transformacional y el fortalecimiento como organización inteligente.

\section{2.- MÉTODO}

\section{Diseño}

La investigación de donde se origina este artículo fue realizada desde un enfoque positivista, con una metodología cuantitativa, de tipo analítica nivel explicativa, dada la definición de Pelekais et al. (2014), y un diseño no experimental, transeccional, de campo. Las variables trabajadas autotomía caudal y cambio organizacional fueron analizadas en un contexto muy específico referenciado a las empresas familiares por departamentos.

\section{Participantes}

La población estuvo representada por empresas familiares de ventas por departamentos en el municipio Maracaibo, que fueron seleccionadas unificando criterios basados en los siguientes aspectos:

(a) Empresas familiares.

(b) Ubicadas en parroquias del municipio Maracaibo (estado Zulia).

(c) Pertenecientes a la categoría de pequeñas y medianas empresas por su tamaño; y para efectos de este estudio se consideraron las ocho (8) entidades que se muestran en el cuadro 1 correspondiente:

Cuadro 1. Distribución de la población de acuerdo a los criterios a, b y c

\begin{tabular}{|c|c|c|}
\hline Total tiendas & Denominación & Gerentes \\
\hline 4 & Chicolandia & 12 \\
\hline 4 & Wendy's & 4 \\
\hline 4 & Acuario & 3 \\
\hline 2 & Iguana Shop & 3 \\
\hline 2 & NY and Co & 3 \\
\hline 1 & Veesca & 2 \\
\hline 1 & Michelle Imports & 1 \\
\hline 2 & Garota & 30 \\
\hline Total: 20 & & 2 \\
\hline
\end{tabular}

Fuente: elaboración propia (2014). 


\section{Instrumentos}

Fueron diseñados dos (2) instrumentos, uno para cada variable, que se aplicaron a una población de treinta (30) gerentes en ocho (8) entidades; dichos instrumentos tuvieron treinta y tres (33) ítems para la variable autotomía caudal y cuarenta y dos (42) para cambio organizacional, ambos con cinco alternativas de escala tipo Likert. La validez fue llevada a cabo por siete (7) expertos en el área y luego se sometió cada instrumento a una prueba piloto conformada por doce (12) sujetos. La confiabilidad de ambos se realizó a través del cálculo del coeficiente alpha Cronbach, obteniendo como resultado 0.864, lo que dejó evidente que eran altamente fiables.

\section{Procedimientos}

Se inició el protocolo de investigación. La segunda fase estuvo constituida por el avance del capítulo I, en donde fue trabajado el planteamiento y formulación del problema, objetivos, justificación y delimitación de la investigación. Durante la tercera fase se procedió a realizar una revisión de los antecedentes y bases teóricas, se planteó el sistema de variables con su respectiva operacionalización, que constituyó el capítulo II. La cuarta fase se orientó al establecimiento del marco metodológico, en donde se habló sobre el enfoque epistemológico, tipo y diseño de la investigación, población, técnica e instrumento de recolección de datos, entre otros. La quinta fase incluyó el análisis y discusión de los resultados, conclusiones, recomendaciones, y finalmente las referencias bibliográficas que soportan el trabajo. Concluida esta fase, se procedió a elaborar el informe final, así como el artículo producto del trabajo realizado.

\section{3.- RESULTADOS}

El tipo de resultado es descriptivo, enfocado a la autotomía caudal como estrategia gerencial para el cambio organizacional en empresas familiares. Los datos obtenidos por los instrumentos aplicados fueron procesados a través del programa SSPS versión 20, considerando la estadística inferencial, y con ella medidas de variabilidad, métodos paramétricos para la comparación de medias con los rangos ponderados en la determinación del grado de presencia de cada indicador, dimensiones y variables objetos de estudio. De igual modo se empleó la técnica de análisis de varianza (Anova) y la prueba post hoc de Tukey.

Se evidenció de manera general que en las etapas de transformación la "situación amenaza" es el primer proceso que enfrentan los gerentes en el proceso de transformación en las tiendas familiares por departamento, seguido de la "renovación", “dolor" y "desprendimiento". 


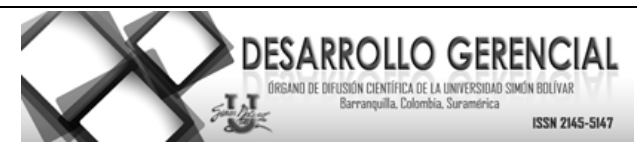

Con respecto a la comparación de la prueba de múltiples rangos post hoc de Tukey, revela las diferencias entre los diversos indicadores de la dimensión etapas de transformación de la variable autotomía caudal, al mismo tiempo que se destaca la homocedasticidad de las varianzas entre sus indicadores (ver tabla 2).

Tabla 2. Dimensión: etapas de transformación

Anova de un factor

Subconjuntos homogéneos

HSD de Tukey ${ }^{\mathrm{a}}$

\begin{tabular}{|l|c|c|c|}
\hline \multicolumn{1}{|c|}{ Factor } & N & \multicolumn{2}{c|}{ Subconjunto para alfa $=\mathbf{0 . 0 5}$} \\
\cline { 3 - 4 } & & $\mathbf{1}$ & $\mathbf{2}$ \\
\hline Desprendimiento & 30 & 3,4560 & 4,0557 \\
\hline Dolor & 30 & & 4,2337 \\
\hline Renovación & 30 & & 4,3333 \\
\hline Situación amenaza & 30 & &, 526 \\
\hline Sig. & & 1,000 & \\
\hline
\end{tabular}

Se muestran las medias para los grupos en los subconjuntos homogéneos.

a. Usa el tamaño muestral de la media armónica $=30,000$.

Fuente: elaboración propia (2014)

En las etapas de transformación analizadas en los contextos estudiados, se evidencia que la situación amenaza forma parte de los aspectos del ambiente que pueden llegar a constituir un peligro para el logro de los objetivos de las empresas familiares, es un acto social o fenómeno natural que se manifiesta en el entorno de las mismas.

Con respecto a los requerimientos de afrontamiento para el cambio organizacional en empresas familiares del municipio Maracaibo del estado Zulia, se destaca que al emplear la técnica estadística análisis de la varianza (Anova) la significancia alcanzada por los indicadores de la dimensión requerimientos de afrontamiento la cual obtuvo un nivel de significancia de 0,000 , siendo este valor menor que $0,05(0,000<0,05$; nivel de significancia referencial); se demuestra que existen diferencias altamente significativas entre los indicadores comparados, y con ello se señala que no todos tienen el mismo grado de presencia dentro de esta dimensión. A continuación se muestra el comportamiento de los sujetos en los contextos investigados (ver tabla 3 ).

\section{Tabla 3. Nivel de significancia (Anova de un factor)}

\section{Requerimientos de afrontamiento}

\begin{tabular}{|c|c|r|r|r|r|}
\hline & $\begin{array}{c}\text { Suma de } \\
\text { cuadrados }\end{array}$ & \multicolumn{1}{|c|}{ gl } & \multicolumn{1}{c|}{$\begin{array}{c}\text { Media } \\
\text { cuadrática }\end{array}$} & F & Sig. \\
\hline Intergrupos & 11,498 & 3 & 3,833 & 11,345 &, 000 \\
\hline Intragrupos & 39,186 & 116 &, 338 & & \\
\hline Total & 50,684 & 119 & & & \\
\hline
\end{tabular}

Fuente: elaboración propia (2014). 
La comparación de la prueba de múltiples rangos post hoc de Tukey revela las diferencias entre los diversos indicadores de la dimensión tipos de fuerza de la variable cambio organizacional, al mismo tiempo se destaca la homocedasticidad de las varianzas entre los indicadores (ver tabla 4).

Tabla 4. Dimensión: requerimientos de afrontamiento Anova de un factor

HSD de Tukey Subconjuntos homogéneos

\begin{tabular}{|c|c|c|c|}
\hline \multirow[t]{2}{*}{ Factor } & \multirow[t]{2}{*}{$\mathbf{N}$} & \multicolumn{2}{|c|}{ Subconjunto para alfa $=0.05$} \\
\hline & & 1 & 2 \\
\hline Liderazgo & 30 & 4,0111 & \\
\hline Participación & 30 & & 4,5333 \\
\hline $\begin{array}{l}\text { Fortalecimiento como organización } \\
\text { inteligente }\end{array}$ & 30 & & 4,7333 \\
\hline Formación & 30 & & 4,8000 \\
\hline Sig. & & 1,000 & 290 \\
\hline
\end{tabular}

Se muestran las medias para los grupos en los subconjuntos homogéneos.

a. Usa el tamaño muestral de la media armónica $=30,000$.

Fuente: elaboración propia (2014).

\section{4.- DISCUSIÓN}

Los resultados acontecidos permitieron establecer semejanzas con lo expresado por Dempsey (2009), quien muestra que con un cambio existe una pérdida y con la pérdida hay duelo como respuesta normal a un dolor. En cuanto al indicador más favorecido por la opinión de los sujetos encuestados, la "situación amenaza" se sitúa con mayor promedio; es decir, consideran que esta se presenta y es importante.

De igual forma, guarda semejanza con lo manifestado por los autores Iglesias y Hernández (2013), de quienes se hace una inferencia de consiliencia, al expresar que los gerentes se sienten en situación de amenaza al momento de sentir que su entorno puede estar comprometido a cambios, sean drásticos o no, pero que requieren de nuevos contextos en los cuales su comportamiento rutinario se ve expuesto a transformaciones. Finalmente, partiendo de estos resultados se concibe que los sujetos informantes muestran coherencia en sus criterios manifestados en las etapas de transformación, lo cual coincide con el planteamiento de Bridges (2009) al mostrar que el cambio comprende en etapas de desprendimiento, que obedecen a factores del entorno para propiciar la transformación y lograr la renovación.

Por otro lado, en la tabla 4 se exponen los resultados de la comparación establecida, donde la prueba de múltiples rangos de Tukey ubicó dos subconjuntos, basados en la similitud y diferencias de comportamiento entre sus medias. El primero de ellos corresponde al indicador liderazgo, que obtuvo la calificación más baja con una media $=4,01$ puntos; seguido del indicador participación, cuya media es $=$ 


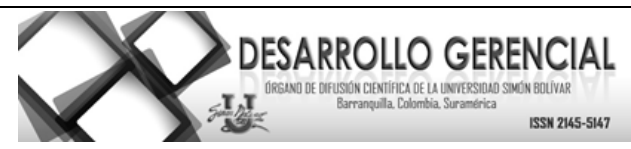

4,53 puntos; seguido del indicador fortalecimiento como organización inteligente, cuya media es $=4,73$ puntos, $\mathrm{y}$, por último, con la media más alta el indicador formación, cuya media es $=4,8$ puntos.

A este efecto se sustenta lo señalado por Benavides Espindola (2001), cuando expresa que precisamente por ser de carácter clave, el desarrollo de una organización es diferente al buscar un cambio gestionado en las empresas donde el desempeño de los procesos de cambio posee un punto importante conociéndose que las estrategias están entre las propuestas de reforma y el logro de una mejora en la práctica. Es por ello que las empresas familiares deben tener la capacidad de decidir sobre los proyectos de cambio de acuerdo con sus propias prioridades; de allí que tienen que existir oportunidades de trabajar juntos para el desarrollo del cambio organizacional, así como los recursos adecuados.

\section{CONCLUSIONES}

Se concluye que según las etapas de transformación en los contextos estudiados, la situación amenaza forma parte de los aspectos del ambiente que puede llegar a constituir un peligro para el logro de los objetivos y permanencia de las empresas familiares, de donde se infiere que es un acto social o fenómeno natural manifiesto en su propio entorno.

Así mismo, los resultados evidencian que es necesario el cumplimiento de estas etapas para darle sentido al mismo proceso de transformación, ya que también se convierte en el elemento que provocaría una respuesta de carácter estratégico a tiempo. En este mismo orden de ideas, se hace mención a las etapas de desprendimiento que involucran el dolor y la transición a un nuevo comienzo que culmina en renovación.

Por consiguiente, es necesario tomar conciencia que las organizaciones actualmente se encuentran en una permanente evolución y que la dinámica de los procesos no se detiene, y esa situación es la que les permite valorar la necesidad y urgencia de asumir la diferencia como parte de la gestión. Por lo tanto, esta transformación facilita evolucionar y dimensionar la gestión; pretender quedarse en el pasado es renunciar a la realidad que plantea el mercado, lo natural de los procesos es que se vaya transitando de un estado a otro. En este sentido, el cambio se usa cuando efectivamente se trata de experiencias cualitativas que llevan a la aparición o incorporación de nuevos elementos que reemplazan a otros.

Finalmente, se recomienda desarrollar talleres de capacitación al personal gerencial sobre manejo apropiado de las etapas de transformación, para que tenga conocimientos sólidos y formales a lo referido a un proceso de desprendimiento correcto, a través del cual puede transformarse el cambio en ganancia y emoción en energía. Esto precisa el manejo apropiado de las situaciones de amenaza, a fin de que se conviertan en gestores y así asumir diferentes posturas actitudinales con las cuales logren explotar sus capacidades hasta niveles superiores u obtener la adaptación que se requiera según los adelantos 
tecnológicos o cambios de faenas como estrategia de rotación tal cual lo inste la gerencia por determinadas razones.

\section{5.- REFERENCIAS}

Albornoz. (2009). Cultura y cambio organizacional en empresas de telefonía móvil. Tesis de Maestría. Maestrìa en Gerencia de Recursos Humanos. Universidad Dr. Rafael Belloso Chacín

Arnold, E. N. (1994). Investigating the origins of performance advantage: adaptation, exaptation and lineage effects. En:EGLETON, P. \& R. I. VANE-WRIGHT (Eds.) Phylogenetics and Ecology. Academic Press, London.

Bellairs, A.D’A. \& Bryant, S.V. (1985). Autotomy and regeneration in reptiles. En C. Gans \& F. Billet (Ed.), Biology of the Reptilia (pp. 301-410). New York: John Wiley and Sons.

Benavides Espindola, O. (2001). Competencias y competitividad: Diseño para organizaciones latinoamericanas. Colombia: McGraw-Hill.

Bridges, William (2009). Managing Transitions: Making the Most of Change, ( $\left.3^{\text {ra }} \mathrm{ed}.\right)$.

Reino Unido: Editorial Nicholas Brealey Publishing.

Cornejo, A. (2004). Complejidad y caos. Guía para la administración del siglo XXI. Recuperado de: http://www. scribd. com/doc/22615328/Complejidad-y-Caos-en-Las-Organizaciones-Ing-AlfonsoCornejo.

Davis, K. \& Newstrom, J. (2003). Comportamiento Humano en el Trabajo. México: Editorial McGrawHill.

Delors, J. (1996). La educación encierra un tesoro. Informe a la Unesco de la Comisión Internacional sobre la Educación para el Siglo XXI. Madrid: Santillana Ediciones Unesco.

Dempsey, K. (2009). Desprenderse de sus enfermedades (4. ${ }^{\text {a }}$ ed.). Phoenix (EE. UU.): Editorial Treypress.

Etkin, J. (2009). Gestión de la complejidad en las organizaciones. Buenos Aires: Editorial Granica.

Gordon, J. (2000). Comportamiento Organizacional. México: Editorial Prentice Hall Hispanoamericana.

Guizar, Rafael (2004). Desarrollo organizacional. Segunda edición. México: Editorial McGraw-Hill.

Iglesias, I. \& Hernández, I. (2013). Enfermedades más comunes en las iguanas. México: Editorial de Vecchi.

Kinicki, A. \& Kreitner, R. (2003). Comportamiento organizacional. México: McGraw-Hill. 


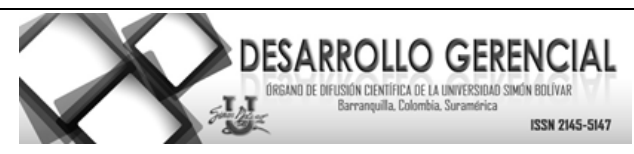

Koontz, H. \& Weihrich, H. (2004). Administración. México: Editorial McGraw-Hill.

Lorenz, K. (2000). Fundamentos de la Etología. (R. Bein Mayer, Trad.). Argentina: Editorial Paidós.

Martín Rueda, J. (1992). Consecuencias ecológicas de la pérdida de la cola en la lagartija serrana (Lacerta Monticola). Tesis doctoral no publicada, Universidad Complutense de Madrid, España.

Morales, M. (2007). Cambio Organizacional y Estrés Laboral en los Gerentes de las Empresas Regionales de televisión por Cable. Tesis de grado no publicada, Universidad Privada Dr. Rafael Belloso Chacín, Maracaibo, Venezuela.

Morin, E. (2007). El conocimiento del conocimiento. Madrid: Ediciones Cátedra.

Pelekais, C.; Seijo, C.; Neuman, N. \& Tromp, L. (2014). El ABC de la investigación. Guía didáctica. Maracaibo: Ediciones Astro Data.

Pelekais, C. \& Soto, M. (2014). El cambio desde las organizaciones caordicas. Maracaibo: Universidad Privada Dr. Rafael Belloso Chacín.

Ponce, R. (2004). Manejo de la Resistencia al Cambio (Un enfoque desde la perspectiva de la psicología organizacional). Julio de 2004.

Prigogine, I. (1993). ¿Tan solo una ilusión? Una exploración del caos al orden. Barcelona: Tusquets.

Robbins, S. (2004). Comportamiento organizacional. México: Editorial Prentice Hall.

Sagarin, R. (2013). Para más adaptables, tomar una lección de biología. Revista de Negocios de Harvard. Obtenido desde https:/hbr.org/2013/03/to-become-more-adaptabletake $+\& \mathrm{~cd}=1 \& \mathrm{hl}=\mathrm{es} \& \mathrm{ct}=\mathrm{clnk} \& \mathrm{gl}=\mathrm{ve}$

Schvarstein, L. (1998). Diseño de organizaciones. Tensiones y paradojas. Buenos Aires: Paidós.

Senge, P. (2000). La danza del cambio: El reto de avanzar en las organizaciones que aprenden. España: Editorial Gestión 2000.

Zani, P.; Russell, A. \& Higham, T. (2013). Integrative Biology of Tail Autonomy in Lizards. Biología integrativa de la autotomía caudal en las lagartijas. Riverside (California): Universidad de California 